\title{
Heap-based algorithm and one-dimensional expanding Universe
}

\author{
Duccio Fanelli ${ }^{1}$, Erik Aurell ${ }^{2}$, Alain Noullez ${ }^{3}$ \\ ${ }^{1}$ Dept. of Numerical Analysis and Computer Science, KTH, SE-100 44 \\ Stockholm, Sweden \\ 2 SICS, Box 1263, SE-164 29 Kista, Sweden \\ ${ }^{3}$ CNRS, Observatoire de la Côte d'Azur, F-06304 Nice Cedex 4, France
}

\begin{abstract}
A fast algorithm for studying one-dimensional systems that are Lagrangian integrable between collisions is presented. The algorithm is based on an event-driven scheme, and uses a heap ordered set of predicted future events. As an application, we discuss the case of an expanding one-dimensional Universe.
\end{abstract}

We consider the non-linear evolution of a one-dimensional perturbation in a three dimensional spherically symmetric Einstein-de Sitter Universe, under the conditions where Newtonian mechanics applies. We focus our attention on a small slice of the whole Universe, formally considering the mutual interaction of $N$ massive sheets (particles) in the expanding medium. By performing both position and time rescaling (respectively, $x_{i} \propto t^{2 / 3} \tilde{x}_{i}$ and $d t \propto t d \tau$ ), one ends up with the following equation of motion for the $i$-th sheet :

$$
\frac{d^{2} x_{i}}{d \tau^{2}}+\frac{\omega_{J 0}}{\sqrt{2}} \frac{d x_{i}}{d \tau}-\omega_{J 0}^{2} x_{i}=\Gamma_{g r a v}^{i}
$$

where $\omega_{J 0} \equiv\left(4 \pi G \rho_{0}\right)^{1 / 2}$ is the Jeans frequency. $\Gamma_{\text {grav }}^{i}$ is proportional to the net mass difference between the right and the left of the given particle, and is therefore constant between collisions. The model (1) was first derived by Rouet and collaborators (Rouet,Feix \& Navet 1990) and has been recently revisited and improved in Aurell \& Fanelli (2001). Numerical simulations are performed by using a version of the heap-based algorithm presented in Noullez, Fanelli \& Aurell (2001). This event-driven scheme is based on the capability of computing the next crossing time between two adjacent particles. The collision time of each particle with its neighbor on the right is given by the smallest real positive root of a cubic equation. The results are stored in an array of size $N-1$, that is then heap-ordered. The minimum value $t_{\min }$, is then the first heap element and is easily selected with a cost of $\mathcal{O}(1)$ operations. The particles $j$ and $j+1$ experiencing this first collision are then evolved up to $t_{\min }$ and their states re-arranged according to the prescriptions of the dynamics (momenta simply exchanged). Further, their next crossing time is computed by solving a quadratic equation, that results by factorizing out the trivial $t_{\text {next }}=0$ solution from the cubic. The two values of their crossing times with their respective nearest neighbors $j-1$ and $j+2$ need also to be updated: this again implies 

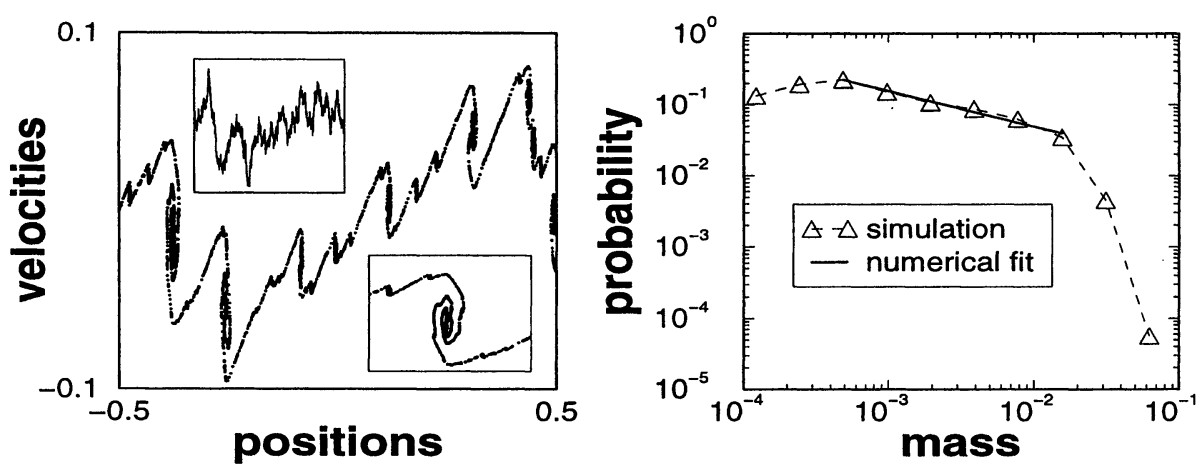

Figure 1. Left plot: Velocity field vs. positions, starting form a single speed Brownian motion initial conditions (upper inset). The lower inset is a zoom of a massive agglomeration. Reflecting boundaries are assumed. The unit of length is the box size. Here $N=16384$ and $\tau=5.0 \omega_{J 0}^{-1}$ or $\left(t=1.9045 \times 10^{3} \omega_{J 0}^{-1}\right)$. Right plot: MOF for Brownian initial velocities at time $\tau=3.3 \omega_{J 0}^{-1}$ (or $t=517.14 \omega_{J 0}^{-1}$ ). Here $N=16384$, and we did 1000 independent realizations.

finding the roots of two cubic equations. The new predicted collision times replace their old values in the heap, which is then rearranged with at most $\mathcal{O}(\log N)$ operations. The whole procedure can then be iterated. In conclusion, the asymptotic complexity of the algorithm is in the worst case $\mathcal{O}(Z \log N)$, where $Z$ is the total number of crossings.

As an example of the results of simulation, we show in Fig. 1 a late time phase space portrait, if the initial velocity is chosen to be a single-speed Brownian motion. Spiral structures are clearly displayed, as well as structured filaments connecting the dense agglomerations. To investigate quantitatively the particles distribution, we introduce the mass octave function (MOF), see Aurell \& Fanelli (2001). MOF measure the probability to find a non zero contribution to the mass density, as function of the mass itself, coarse-grained in octaves. The right plot of Figure 1 shows, in log-log scale, the MOF computed for an intermediate stage of evolution. In a finite range of the mass support, the MOF decays as a power law with exponent -0.5. This result is in agreement with She, Aurell \& Frisch (1992), where in the framework of the adhesion model (i.e. Burgers' equation) the number density per unit length of shock locations holding mass $m$ is shown to be distributed as a power law $m^{-1-h}$.

\section{References}

Aurell, E., \& Fanelli, D. 2001, submitted to Phys. Rev. E, cond-mat/0106444

Noullez, A., Fanelli, D., \& Aurell E. 2001, submitted to JCPh, cond-mat/0101336

Rouet, J.L., Feix, M.R., E Navet, M. 1990, Vistas in Astronomy, 33, 357

She, S.-Z., Aurell, E., \& Frisch U. 1992, Comm. Math. Phys., 148, 623 